\title{
Avant-propos
}

\section{Pratiques et réflexions autour des dispositifs d'apprentissage et de formation des communicateurs}

\author{
Valérie Lépine, maître de conférences en \\ sciences de l'information et de la communication, \\ Université Grenoble Alpes, Gresec, \\ valerie.lepine@iut2.upmf-grenoble.fr \\ Marc D.David, professeur, \\ Université de Sherbrooke, \\ marc.d.david@usherbrooke.ca
}

Le Réseau international sur la professionnalisation des communicateurs (RESIPROC) s'est donné pour ambition d'identifier, structurer et partager des recherches, des connaissances mais aussi des expériences ou des pratiques professionnelles relatives aux évolutions des industries des communications et de leurs acteurs. Ce secteur est soumis à des changements et à des recompositions rapides : émergence de nouveaux métiers, technicisation des dispositifs, évolution des formes de régulations info-communicationnelles, organisationnelles et sociales, repositionnement des acteurs en présence, etc.

La dispersion en de nombreux domaines ou activités des métiers de la communication invite à réinterroger le socle des compétences communes et des compétences spécialisées nécessaires à l'exercice de ces métiers. En dépit des spécificités sectorielles (secteur humanitaire, associatif; secteur des institutions publiques ou gouvernementales ; secteur marchand) ou techniques (événementiel, édition, web, etc.) quelles lectures et quelles structurations en termes de dispositifs de formation peut-on faire des acquisitions ou des postures théoriques, méthodologiques et pratiques qui contribuent au professionnalisme des acteurs en charge des communications dans les différents types d'organisations ? Le travail de structuration de la professionnalisation à travers des dispositifs de formation est-il 
(et doit-il être) élaboré en lien avec celui de définition des référentiels de métiers auxquels les employeurs font appel pour définir les missions qui seront confiées aux futurs professionnels?

Toutes ces questions invitent à penser l'articulation, dans un domaine particulièrement exposé à des évolutions incessantes, entre pratiques professionnelles et formation.

Cette seconde édition des Cahiers du RESIPROC est consacrée aux enjeux, aux modalités d'apprentissage et aux conséquences de la formation des communicateurs. Les articles qui la composent ont été sélectionnés pour être enrichis et retravaillés parmi l'ensemble des contributions présentées au colloque international «Entre réflexif et prescriptif: Analyse des dispositifs d'apprentissage et de formation des communicateurs » qui s'est tenu dans le cadre du $81^{\text {ime }}$ Congrès de l'ACFAS les 9 et 10 mai 2013 sous la responsabilité scientifique de Marc D. David et Valérie Lépine. Ce numéro est organisé autour de deux thématiques principales : la première est centrée sur les aspects entourant la structuration de la formation et de la pédagogie entourant l'enseignement de certains champs disciplinaires de la communication; la seconde porte sur les dynamiques de consolidation et de légitimation des compétences et sur leur contribution au professionnalisme des communicateurs.

\section{Quels dispositifs de formation et quelle pédagogie en communication?}

Les dispositifs pédagogiques qui prétendent à la professionnalisation en communication ont pris des formes multiples tant dans les filières technologiques les plus anciennes - qu'académiques. À partir de l'hypothèse, pas toujours explicitée, que certains apprentissages se développent davantage dans des processus d'imprégnation/expérience que dans des processus de transmission/acquisition, les formations ont organisé des dispositifs de " mise en contact » (sous la forme de stage, d'alternance, de commande ou de participation des professionnels aux enseignements, etc.) avec les environnements organisationnels et les acteurs professionnels. D'autres initiatives innovantes, liées aux TIC et/ou aux usages des réseaux socionumériques, prennent pour support pédagogique les outils ou dispositifs d'information et de communication qui sont utilisés par les professionnels dans leurs activités.

Les formations à visée professionnelle en communication peuvent être étayées par une véritable réflexion sur la traduction en objectifs et en compétences des programmes pédagogiques. Elles sont le plus souvent largement co-construites dans des interactions impliquant dans des rôles distincts mais complémentaires professionnels et académiques. Les contributions de cette première partie en témoignent largement. 
Raphaëlle Bats, Marie-France Peyrelong et Martine Vila-Raimondi sont toutes trois enseignantes et responsables de formations de niveau master préparant de futurs cadres des métiers de l'information et de la communication, d'une part au sein de l'école nationale supérieure des sciences de l'information et des bibliothèques (enssib) et d'autre part à l'Université Lyon 3. Elles montrent que les référentiels qui existent en France pour les métiers des bibliothèques, de la documentation ainsi que pour certains métiers de la communication mobilisent une sémantique et des descriptions très génériques qui laissent une large part d'interprétation tant aux formateurs qu'aux employeurs ou aux professionnels. Dépasser les limites des descripteurs de l'activité et conduire les étudiants vers la compréhension et l'appropriation de significations et d'actions partageables et pertinentes dans le cadre concret des pratiques professionnelles, constitue un véritable défi. Il s'agit rien moins que de faire «entrer le réel » dans des apprentissages professionnalisant les étudiants tout en favorisant leur réflexivité et leur autonomie. Les auteures nous livrent quelques pistes pédagogiques explorées dans différents dispositifs de formation : speed meeting, film pédagogique, mise en situation.

La contribution d'Anne-Marie Cotton (Arteveldehogeschool Univeristy College Gent) permet d'éclairer sous un autre angle le rapport entre référentiels-métiers et construction pratique d'une pédagogie visant à l'atteinte des compétences prescrites. Dans la filière académique belge considérée, les étudiants du cursus publicité et média planning doivent en troisième année de formation être en capacité d'élaborer un audit de marque (brand review) du type de ceux réalisés en agence conseil, assorti de recommandations stratégiques. L'auteure s'appuie sur une rechercheaction menée sur plusieurs années et dévoile les étapes successives du dispositif pédagogique qui s'est progressivement enrichi ainsi que la complexité des interactions entre apprenants, experts professionnels et enseignants. Elle montre que le chemin d'apprentissage s'inscrit dans une série de médiations impliquant une véritable « casuistique coopérative tuteurée » et des ajustements étroits, individualisés entre étudiants, encadrants professionnels et l'enseignant.

La coopération entre professionnels et universitaires et la formation d'une véritable équipe dédiée au projet d'ingénierie de formation en communication sont aussi au cœur de l'article de Valérie Larroche (Université Lyon 3). L'auteure mobilise le cadre théorique de la traduction (Callon, 1986) pour mettre en perspective l'émergence d'un collectif dont les intérêts croisés et l'implication permettront - malgré les difficultés administratives - la création d'une licence professionnelle qui répond aux besoins du secteur émergent à la fin des années 2000 du marketing digital et des stratégies de gestion des communication et contenus numériques. Ce retour d'expérience permet de mettre en lumière les phases de cette création de formation depuis la première impulsion du projet (primum movens) jusqu'à son aboutissement en montrant que loin d'être linéaire et attribuable à une 
catégorie d'acteurs unique, le processus relève d'enrôlements et leaderships distribués entre professionnels et universitaires.

Bertrand Labasse (Université d'Ottawa) propose d'analyser avec une très grande finesse les contenus des manuels en didactique de la communication. Son étude repose sur un inventaire des préceptes et connaissances opératoires dans une cinquantaine de manuels de communication écrite qui ont en commun de former in fine à une compétence-clé mise en avant par les praticiens de la communication, celle de « la diffusion de représentations au moyen de discours adaptés aux publics visés». Il analyse la structuration des discours pédagogiques pour dégager une catégorisation qui rend plus lisibles - derrière l'apparente diversité des manuels quelques grandes structures-types (approche par genre de documents, approche procédurale, linguistique, etc.) dont l'auteur souligne un grand nombre de limites. La réflexion, transposée aux enseignements académiques en communication, conduit Bertrand Labasse à envisager " les problèmes pédagogiques comme des problèmes épistémologiques » en adoptant une posture plus distanciée qu'instrumentale.

Les enquêtes menées à l'initiative des associations corporatives ou d'organismes institutionnels révèlent l'élévation progressive du niveau de qualification des professionnels de la communication au cours des vingt dernières années. Le niveau de formation initiale est le plus fréquemment au-delà du grade de licence. En revanche, les filières diplômantes sont hétérogènes et les cursus en sciences politiques, en études commerciales et marketing, en gestion et management, alimentent fréquemment la formation des communicateurs qui ne sont pas toujours loin s'en faut - spécifiquement formés à la communication. Les formations actuelles sont-elles des ressources permettant aux jeunes diplômés de s'insérer dans les organisations, de prendre en charge les activités de communication et d'occuper des fonctions reconnnues?

L'article de Zeineb Touati (Université du Havre) et Cristina Badulescu (docteure, Université de Rouen) constitue une transition entre la première partie consacrée aux dispositifs pédagogiques et la seconde partie de ce numéro qui explore sous différents angles les processus de formation et de transformation des professionalismes au sein même de l'activité et des organisations. Ces auteures questionnent le rapport entre niveau de diplôme, compétences, insertion professionnelle et mobilité dans la trajectoire des jeunes diplômés en communication dans le contexte français. Le système d'enseignement post-baccalauréat en France présente des spécificités et notamment celle - séculaire - d'une forte partition entre universités et grandes écoles qui renforce les inégalités sociales d'accès aux carrières. Malgré des efforts considérables pour développer une offre de formation professionnalisante de qualité intégrée au tissu économique, les filières universitaires françaises pâtissent d'un déficit d'image auprès des entreprises. La socialisation professionnelle (à travers les stages), les modalités d'accompagnement 
d'une construction identitaire (développée dans les études de cas, projets tutorés et autres mises en situation) organisées de manière systématique dans les filières infocom des instituts universitaires technologiques (IUT) ne suffisent pas à garantir l'employabilité des diplômés de licence en communication face à la concurrence des diplômés grandes écoles en commerce, marketing et management. Ces derniers préemptent les postes d'encadrement des métiers de la communication au détriment des titulaires de masters et obtiennent des rémunérations largement supérieures alors même qu'ils n'ont pas de formation approfondie et de compétences particulières voire minimales - dans le domaine des communications. Il y a donc, dans certains contextes semble-t-il, une faible corrélation entre compétences validées par un diplôme universitaire en communication et trajectoire professionnelle dans les métiers de la communication en France.

La seconde partie de ces Cahiers du RESIPROC tente d'apporter des éclairages sectoriels et catégorielles plus précis sur les compétences, les métiers et professionnels qui les exercent.

\section{2 . Les compétences professionnelles des communicateurs : des métiers en redéfinition et en quête de légitimation}

Les dynamiques de consolidation, de pérennisation et de légitimation de leurs compétences semblent être au cœur des préoccupation des communicateurs et ce, dans tous les sphères d'activitités professionnelles que ce soit dans l'analyse d'une culture créative (voir Baillargeon), sur la position des communicants internes (voir Brulois, Charpentier et Viers) ou sur les défis relevés chez référenceurs (voir Domenget et Michel).

Dany Baillargeon (Université de Sherbrooke) se penche sur une définition opératoire de la culture créative des agences de publicité. À l'aide d'une étude de cas, l'auteur illustre certains pans de la culture créative d'une petite agence de publicité québécoise en région et vise à savoir comment cette culture créative participe à faire circuler l'apprentissage « de qui est » et « de ce qui est » créatif. Cette étude de cas suggère une mise en relation « de la notion de culture créative avec celle d'apprentissage et de transmission du savoir créatif ». Selon Dany Baillargeon, la performativité de l'ethos créatif des employés comme celle de l'agence participent à maintenir cette culture créative autant qu'elle transmet des formes d'apprentissage créatif à travers des dynamiques identitaires. De plus, sa recherche souligne la présence d'une éducation créative continuellement à faire - et à refaire - auprès des clients par « l'utilisation de structures de légitimation interne et externe à l'agence de publicité».

Vincent Brulois (Université Paris 13, Sorbonne Paris Cité), Jean-Marie Charpentier (ACFI) et Jacques Viers (APSE) se sont interrogés sur la position des 
communicants internes à travers deux expériences de travail. Selon les auteurs, pardelà les compétences, les savoir-faire professionnels classiquement requis ou sollicités, des communicants témoignent d'un intérêt nouveau pour des savoirs, des connaissances en sciences humaines et sociales ainsi que pour des démarches réflexives de type analyse de pratiques. Selon eux, «l'entreprise est en tension » car le changement y est continu. Partant des situations de travail des communicants, les auteurs proposent de pousser plus avant la réflexivité sur leurs pratiques, de les aider à comprendre leurs logiques d'action, à se mettre à distance et finalement, d'étayer une posture professionnelle différente. Dans ce contexte, les auteurs présentent les sciences humaines et sociales (SHS) comme « un moyen pour des professionnels de comprendre, de décoder, de trouver du sens par eux-mêmes » toujours dans le cadre d'une démarche réflexive permettant aux communicants de définir leur propre posture professionnelle.

Jean-Claude Domenget (Université de Franche-Comté) et Jean-Luc Michel (Université de Saint-Etienne) proposent de montrer comment le récent métier de référenceur s'inscrit dans la structuration générale des professions de la communication à travers une recherche de validation de la pérennisation du métier au travers des diversifications et des recompositions des tâches autour d'un noyau invariant de compétences. Ensuite les auteurs, se penchent sur le processus de légitimation permettant aux référenceurs d'accéder, ultimement, aux décisions stratégiques de communication. Pour cela, les auteurs proposent une modélisation dynamique autour de cinq profils type de référenceurs (Défricheurs, Explorateurs, Organisateurs, Planificateurs, Stratèges) Les auteurs analysent à travers leur étude " le long cheminement vers la légitimité du référencement en montrant l'élargissement de ses tâches opérationnelles du début à des missions de plus en plus stratégiques et globales ». En somme, le référencement viserait à franchir une nouvelle étape, " celle de gagner sa légitimité, c'est-à-dire d'être jugé assez crédible pour participer aux choix stratégiques en amont de la communication par le web».

\section{3. «Points de vue » sur la professionnalisation des communicateurs}

Nous proposons dans cette nouvelle section de donner un espace aux propos, essais, voire pamphlets permettant aux chercheurs et praticiens de livrer leurs perspectives particulières sur tous les aspects reliés à la professionnalisation des communicateurs.

Bernard Dagenais (Laval) se livre à une charge virulente envers l'utilisation abusive et mensongère de stratégies de responsabilité sociale d'entreprise (RSE) par différentes entreprises afin de se doter " d'une image de générosité sur la place publique sans trop se soucier de son véritable comportement ». À travers une série d'exemples, l'auteur s'attaque particulièrement au détournement de sens d'une 
véritable RSE axée sur les actions concrètes visant à améliorer le bilan social de l'entreprise au détriment de discours trompeurs essentiellement utilisés pour voiler des activité douteuses. Dans cette perspective, la RSE devient « un instrument d'image avant tout ». L'entreprise l'utilise afin de mieux cultiver son statut de bon citoyen corporatif et toujours selon l'auteur, « les activités de RSE sont choisies d'une part en fonction des zones sensibles de l'entreprise dans l'opinion publique et d'autre part en fonction des retombées économiques attendues ». En somme, les critiques de l'auteur ouvrent la voie à la nécessité de responsabiliser les comportements éthiques et déontologiques des praticiens dans tous les sphères de l'organisation et particulièrement des professionnels en charge des discours d'entreprise (corporate) ou de la communication marketing. 